\title{
Application Program Freezes, Becomes Nonfunctional
}

National Cancer Institute

\section{Source}

National Cancer Institute. Application Program Freezes, Becomes Nonfunctional. NCI

Thesaurus. Code C133578.

Problem associated with freezing and becoming nonfunctional of an application

program. 